\title{
MEJORANDO LA SALUD HUMANA: ENFOQUE DE PROMOCIÓN DE LA SALUD, PREVENCIÓN DE LA ENFERMEDAD Y EDUCACIÓN EN SALUD
}

\author{
IMPROVING HUMAN HEALTH: FOCUS ON HEALTH PROMOTION, \\ DISEASE PREVENTION AND HEALTH EDUCATION \\ Miguel A. Pérez', Luz Marina-Alonso², Cassie Valencia', Olga Caucil-Garcia²
}

\begin{abstract}
RESUMEN
Objetivos: Reflexionar sobre los avances de la promoción de la salud y prevención de la enfermedad, la educación en salud y los retos en la formación de los estudiantes en medicina y salud publica en América Latina. Métodos: Se realizó revisión de bases de datos sobre promoción de la salud, prevención de la enfermedad, educación en salud, educación y formación en medicina, atención primaria en salud, se tomó las últimas dos décadas para llegar a conclusiones diseñadas a fortalecer la formación educativa de médicos y especialistas en promoción de la salud. Resultados: La promoción de la salud y prevención de enfermedad y el componente educación han tenido legado de distintas declaraciones y conferencias internacionales y han definido estrategias y acciones claras que van desde acciones intersectoriales y un compromiso en las instituciones formadoras de recursos en salud. La educación en salud ha fortalecido los programas de promoción y prevención de ahí que el reto de instituciones directivas de la salud y formadoras de recursos humanos en salud han trabajado guías y materiales educativos que han sido considerados fundamentales para el trabajo con los determinantes sociales de la salud. Conclusiones: En esta revisión se destaca el aporte de los países para el trabajo en promoción de la salud y prevención de la enfermedad, dejando claro el esfuerzo que realizan instituciones rectoras de salud y formadoras de recursos para fortalecer los currículos educativos, sin embargo se requiere continuar con este tipo de iniciativas para fortalecer los currículos de salud.
\end{abstract}

Palabras clave: Promoción de la salud; Prevención de la enfermedad; Educación en salud; Atención Primaria en Salud. (fuente: DeCS BIREME)

\begin{abstract}
Objective: To reflect on advances of health promotion and disease prevention, health education and the challenges in the training of medical and public health students in Latin America. Methods: A review of databases on health promotion, disease prevention, health education, medical education and training, and primary health care was undertaken to reach conclusions designed to strengthen the educational training of physicians and health promotion specialists. Results: International health promotion and disease prevention conferences have provided clear strategies and actions designed to improve the health status of individuals around the world. In this article we explore the need to better integrate health promotion and disease prevention strategies in the training of health care professionals. Conclusions: This review highlights the need to strengthen educational curricula to reduce health disparities.
\end{abstract}

Key words: Health Promotion; Disease Prevention; Health Education; Primary Health Care. (source: MeSH NLM) 


\section{INTRODUCCIÓN}

Tradicionalmente, la medicina occidental se enfoca en tratar y curar los síntomas descritos por los pacientes manteniendo así la calidad de vida del individuo. La salud humana no existe en un vacío, pero representa las realidades socio económicas, políticas, y ambientales del individuo, estos determinantes de la salud representan factores que median entre las inequidades que se derivan de los determinantes estructurales y los resultados de salud (determinantes intermedios), entre ellos se identifican condiciones materiales de vida, psicosociales, de comportamiento, factores biológicos y relacionados con los sistemas de salud y atención sanitaria'. De otra parte, en el mismo sentido la Organización Mundial de la Salud (OMS) provee un marco teórico muy amplio en torno a los determinantes de salud los cuales incluyen la paz, la vivienda, la educación, el acceso a alimentos y agua limpia, los ingresos, el ecosistema sostenible, los recursos sustentables, la justicia social y la equidad ${ }^{2}$

Datos promulgados por la Organización Mundial de la Salud (OMS) indican que durante los últimos 100 años las enfermedades infecciosas tales como la neumonía y la tuberculosis que fueron las principales causas de morbilidad y mortalidad a principios del siglo 20 han sido reemplazadas por enfermedades no transmisibles tales como las enfermedades cardíacas y el cáncer ${ }^{3-7}$. Los datos de la OMS muestran que con 7,4 millones de muertes, la cardiopatía isquémica, una condición crónica no transmisible que puede estar relacionada con las elecciones conductuales de una persona, fue la principal causa de muerte en todo el mundo en $2012^{8-9}$. Este cambio indica una necesidad de re-enfocar las prioridades sanitarias del tratamiento a la promoción de la salud y prevención de las enfermedades.

La promoción de la salud según la Carta de Ottawa (1986), se comprende como "el proceso de habilitar a las personas para incrementar el control y desarrollo de su salud, se mueve más allá de un enfoque en el comportamiento individual e incluye una amplia gama de intervenciones sociales y ambientales"10-11. La promoción de la salud incluye "un proceso político y social que abarca no solamente las acciones dirigidas directamente a fortalecer las habilidades y capacidades de los individuos, sino también las dirigidas a modificar condiciones sociales, ambientales y económicas, con el fin de mitigar su impacto en la salud pública e individual ${ }^{12}$. En resumen, la promoción de la salud es una intervención social mediante la participación y la educación de las personas en diversos temas de salud con el fin de enseñar a tomar decisiones positivas e informadas para mejorar su calidad de vida ${ }^{10,13}$ y prevenir el desarrollo de patologías que requieran atención médica.

La implementación de programas de promoción de la salud y prevención de la enfermedad corresponde a retos encontrados por los sistemas de atención primaria desarrollados como resultado del Plan Beveridge, el cual planteó los fundamentos para un sistema nacional de cobertura en salud en todos los niveles de atención basado en atención primaria provista por salubristas ${ }^{2,14}$. Cabe recordar a actores que beneficiaron con sus aportes a la promoción de la salud entre ellos a Sigerist quien en la cuarta década del siglo pasado inicia corrientes de medicina social en los Estados Unidos y su constante preocupación por la promoción, se considera que fue el primero en darle ese nombre, entre algunos de sus artículos se destaca Medicine and human Welfare y para la década séptima, el reporte del Ministro de Salud de Canadá, denominado como el Informe Lalonde, el cual cuestiona en forma contundente el enfoque en atención primaria como la primordial forma de mantener la salud poblacional y argumenta que el medioambiente y los estilos de vida son igualmente importantes en los esfuerzos de la salud pública ${ }^{15}$.

En la historia de la promoción de la salud se encuentra que muchos han sido los hechos históricos e iniciativas que han contribuido al desarrollo y fortalecimiento de una red de salud pública diseñada a prolongar no solamente la longevidad humana, pero también la calidad de vida de las personas. El énfasis de la promoción de la salud y la prevención de la enfermedad se observa en el cambio paulatino de comportamientos individuales basados en conocimientos que permitan obtener el nivel óptimo de vida.

Entre los primeros movimientos internacionales realizados sobre este tema se encuentra la celebración realizada en Alma Ata (República de Katzajazan) en 1978, la cual reitera firmemente que la salud es (a) el estado de completo bienestar físico, mental y social, y no solamente la ausencia de afecciones o enfermedades, (b) es un derecho humano fundamental, (c) que el logro del grado más alto posible de salud es un objetivo social sumamente importante en todo el mundo y exige la intervención de muchos otros sectores sociales y económicos, además del de la salud, y finalmente (d) el pueblo tiene el derecho y el deber de participar individual y colectivamente en la planificación y aplicación de su atención de salud ${ }^{16}$.

Unos años mas tarde, la Carta de Ottawa reitera que "La salud no concierne exclusivamente al sector sanitario, 
no se puede separar la salud de otros objetivos, la participación multisectorial debe ser activa, implica el desarrollo de nuevas actitudes que permitan la reorientación de los profesionales y servicios sanitarios como también los modelos y las visiones, la reorientación indiscutiblemente deberá acompañarse de la participación real en la toma de decisiones por los distintos sectores y la gente misma, si se quiere hacer una fuerte alianza a favor de la salud"17.

La Carta de Ottawa identifica tres estrategias básicas para la promoción de la salud incluyendo la abogacía por la salud con el fin de crear las condiciones sanitarias esenciales antes indicadas; facilitar que todas las personas puedan desarrollar su completo potencial de salud; y mediar a favor de la salud entre los distintos intereses encontrados en la sociedad ${ }^{16}$, a este esfuerzo internacional le sigue una serie de conferencias de las cuales destacaremos algunas que de las que se llevaron a cabo en las dos últimas décadas(1995-2015) tales como la declaración de Yakarta, las conferencias de México, Bangkok, Nairobi, Helsinki y la conferencia de Shanghái que se tornan en acciones secuenciales y complementarias, mayor compromiso político, la necesidad de trabajo en salud en todas las políticas y el énfasis en la promoción, como se destaca en la más reciente conferencia².

La Declaración de Yakarta realizada en Indonesia (1997) identifica cinco prioridades para la promoción de salud en el siglo XXI: (a) promover la responsabilidad social para la salud; (b) incrementar las inversiones para el desarrollo de la salud; (c) expandir la colaboración para la promoción de la salud; (d) incrementar la capacidad de la comunidad; y (e) el empoderamiento de los individuos, garantizar una infraestructura para la promoción de la salud.

La conferencia de México en el 2000 nuevamente retoma los campos de acción que se habían discutido en Ottawa y llamó la atención a los políticos centrados en el debate y operar un plan de acción práctico por el cual sentó compromisos. Igual el aporte de la conferencia de Bangkok realizada en Tailandia, resalta compromisos: a) la promoción debe ser componente de toda agenda de gobiernos y responsabilidad en todos los niveles incluyendo el sector empresarial. La conferencia de Nairobi celebrada en Kenia en el 2009. con su llamado a la acción para cerrar la brecha de implementación en la promoción de la salud y fortalecer liderazgo y fuerza de trabajo, seguir situando a la promoción como línea central de trabajo, ampliar el empoderamiento en procesos participativos, aquí la educación en salud es bandera para conseguir estos objetivos.
En el 2013, la Conferencia de Helsinki requiere el desarrollo de políticas que generen un enfoque de trabajo intersectorial, este llamado es reiterado en la Conferencia de Shanghái 21 (2016) la cual sigue reconociendo que la salud y el bienestar son fundamentos para el desarrollo sostenible, aquí se discute la importancia de promocionar medidas que favorezcan el cumplimiento de los Objetivos de Desarrollo Sostenible (ODS), donde se considera que la buena gobernanza es esencial por lo que se exige un mayor compromiso político, aquí el rol de la promoción de la salud fue mayormente enfatizado y la importancia de la educación es importante para sensibilizar a los distintos funcionarios que coordinan las diferentes instituciones de gobierno.

A pesar de esta serie de conferencias internacionales diseñadas a codificar la ciencia y la práctica de la promoción de la salud y el apoyo que las mismas realizan para el cumplimiento de las recomendaciones de la Comisión de los Determinantes Sociales de la salud de la OMS y el aumento de oportunidades en función de la disminución de enfermedades evitables, aun se observa que la promoción sigue siendo marginalizada en muchos países como resultado de mínima inversión económica de parte de los gobiernos y el sector privado; continua siendo vigente el enfoque de la promoción y prevención vis-avis tratamientos médicos, la falta de reglamentos internacionales rigiendo el entrenamiento en estas área, y un mal enfoque en relación a los resultados esperados en promoción, dado que se espera ver resultados inmediatos, los cuales no siempre son evidentes en las estrategias ya que estas están diseñadas a efectos a largo tiempo ${ }^{18-20}$.

\section{Educación para la Salud}

Una estrategia clave teorizada en el trabajo de promoción de la salud y prevención de la enfermedad es la educación en salud, la gente aprenderá a tomar decisiones más saludables para evitar el desarrollo de enfermedades crónicas que puedan ser mortales y costosas. La educación para la salud ha sido definida como "Cualquier combinación de experiencias de aprendizaje planificadas usando prácticas basadas en la evidencia y/o teorías de sonido que brinden la oportunidad de adquirir conocimientos, actitudes y habilidades necesarias para adoptar y mantener comportamientos saludables" ${ }^{\prime 21}$. La educación para la salud centra sus esfuerzos en enseñar a las personas y las comunidades sobre temas de salud para que puedan hacer cosas bien informadas sobre su salud ${ }^{22-24}$. La promoción de la salud y la educación en la salud se enfrentaron a un debate en relación 
al papel que desempeña cada una y esto conllevó a la relevancia de la educación de la salud para el cumplimiento de la promoción ${ }^{13}$.

En relación a la relevancia de la Educación en Salud, para 1999, la OMS emitió una serie de materiales de consulta tales como el "Manual sobre educación sanitaria en atención primaria de salud." Este manual aun hoy es considerado como una las aportaciones más importantes para la implementación de programas de educación para la salud a nivel mundial. Otro recurso sobre este tema es la publicación titulada Introduction to Health Education publicado por el Ministerio de Salud de Etiopia ${ }^{25}$. La intención de estos materiales es ofrecer alternativas para apoyo a la educación para que se pueda trabajar en aportes en función de la promoción de la salud, la prevención de la enfermedad bajo el enfoque de determinantes sociales de la salud.

\section{Promoción de la salud, Prevención de la enfermedad y Medicina.}

Los modelos integrales de atención en salud trabajados en algunos países en América Latina hacen énfasis en la Atención Primaria en Salud donde existe un compromiso a mejorar la salud humana resultando en empoderamiento real de los usuarios de servicios de salud, de igual manera se requiere trabajos continuos en referencia a la promoción de la salud impartida en las escuelas formadoras de recursos, dado que según estudios conservan debilidad ${ }^{26}$.

Los cambios en los paradigmas de salud humana plantean la necesidad desde las instituciones de ofrecer nuevas prácticas a los estudiantes de salud la exigencia del trabajo que requiere los enfoques de atención primaria orientada a la comunidad y la necesidad de regreso al pasado a la atención primaria en salud con enfoque renovada, demanda un fuerte trabajo en la formación médica en general del equipo de salud donde el componente de educación en salud es fundamental ${ }^{27-29}$.

Las escuelas formadoras de recursos en sus niveles de pregrado tienen hoy en día oportunidad de retroalimentar su currículo con la experiencia de las especializaciones relacionadas con las áreas de promoción de la salud y prevención de la enfermedad, entre otras: salud pública, especializaciones en salud familiar, educación en salud etc, conducidas por el norte de renovación de la atención primaria en salud, se puede mencionar el caso de Colombia donde se está trabajando un modelo integral de atención en salud con enfoque de Atención primaria en salud renovada y con un fuerte trabajo en promoción de la salud y prevención con enfoque diferenciado en la atención en los servicios de salud, todo esto se sintoniza con los currículos de formación en salud y con las experiencias a las cuales los estudiantes en formación están expuestos conservando un mayor peso la promoción de la salud. El trabajo con este tipo de modelo en los servicios de salud contribuye al compromiso con los determinantes de salud ya que los mismos constituyen un determinante de salud y aquí la promoción de la salud es bandera ${ }^{30}$.

De acuerdo a la Escuela de Salud Pública en Harvard, las disciplinas de medicina y salud pública buscan mejorar las condiciones de salud humana (ver Tabla 1). Promulgamos que los futuros profesionales en medicina y salud pública pueden beneficiarse de la incorporación de la promoción de la salud en las técnicas de educación sanitaria.

Un proyecto reciente ha sido presentado en la Escuela de Medicina de la Universidad Ludwig Maximilian de Munich, donde 10 estudiantes de medicina humana, ocho estudiantes de salud pública y dos estudiantes de epidemiología tomaron una clase de verano que trataba de la promoción de la salud y prevención de la enfermedad. Las clases se planeaban donde un estudiante de medicina trabaja con un estudiante de salud pública o epidemiología ${ }^{31}$. Cada estudiante recibió una encuesta pre y post sobre su conocimiento de la promoción y prevención de la salud, junto con entrevistas realizadas por la facultad de la utilidad de esta clase ${ }^{31}$. El piloto de esta clase fue visto con mucho éxito, por ejemplo, las encuestas indicaron que el conocimiento de la promoción de la salud y la prevención ha aumentado con todos los estudiantes y profesores que sostuvieron las entrevistas, encontró un trabajo en equipo positivo con las parejas de estudiantes y la motivación para trabajar juntos en proyectos futuros ${ }^{31}$. Si las clases como ésta, se agregaran al currículo de todas las escuelas de medicina, entonces los médicos y otros profesionales interdisciplinarios, junto con los educadores de la salud podrían consultar a los pacientes sobre cómo prevenir enfermedades en lugar de darles medicamentos costosos cuando los síntomas ocurren.

El ejemplo anterior enfatiza los beneficios reales y percibidos resultantes de la incorporación de la promoción de la salud en los currículos de medicina humana, hecho que es importante ya que los comportamientos que contribuyen al cambio de las principales causas de morbilidad y mortalidad requieren que los profesionales de medicina y salud pública unan esfuerzos para aminorar los efectos 
dañinos resultantes de comportamientos insalubres se pueden cambiar para conseguir mejoras en los resultados de salud humana. En la actualidad la promoción de la salud y prevención de la enfermedad en diversos temas de salud, se está enseñando y promoviendo en el campo de la medicina a través de educadores de la salud en lugar de médicos, en especial por el importante beneficio que aportaría a la situación de salud en las Américas ${ }^{32}$.

Los profesionales de la salud tienen un tiempo asignado con sus pacientes el cual podría ser aprovechado de manera máxima en educación preventiva no solo en resolver la medicación para el tratamiento. La mayoría de las enfermedades crónicas pueden ser prevenidas por los pacientes que toman decisiones saludables como comer alimentos saludables y agregar actividad física a sus rutinas diarias ${ }^{9,11}$. A veces es necesario recordar a los pacientes que tomen decisiones saludables o intervengan para cambiar sus comportamientos personales. Los educadores de la salud pueden hacer trabajo tanto de promoción de la salud como de prevención de la enfermedad, para esto es necesario la ayuda de médicos y personal que constituye el equipo de salud integral. Las esperanzas en el trabajo en promoción en salud y educación en salud son cada vez mayor y hoy en día en muchos países de América Latina se llevan proyectos investigativos financiados por instituciones rectoras de la Ciencia y la Tecnología, muchos de estos provechos han priorizado trabajos relacionados con enfermedades crónicas no transmisibles, donde el componente de Educación en salud es fundamental, estos proyectos tienen la ventaja de ser conocidos por los estudiantes de salud e incluso participar de los mismos. Promover la educación en salud en los pacientes contribuye a disminuir las tasas de enfermedad y sus condiciones en deterioro del bienestar y salud igualmente ayuda a una mejor utilización de lo que se invierte en salud en los países para ejemplificar países de américas del sur tienen un porcentaje de inversión del PIB entre 5-10\% ${ }^{33}$.

Tabla 1. Comparación de la Medicina y la Salud Pública.

\section{Medicina}

\section{Salud Pública}
Ética
Primario en la persona
Servicio público
Énfasis
Diagnóstico, tratamiento y atención de la
enfermedad para cada paciente
Prevención de enfermedades y promoción de la salud para toda la comunidad

Énfasis predominante en la atención médica. Sistema uniforme para certificar a especialistas más allá del grado médico profesional
Emplea un espectro de intervenciones dirigidas al medio ambiente, el comportamiento humano y el estilo de vida, y la atención médica
Ciencias sociales

Ciencias numéricas
Tienden a ser una parte electiva de la educación médica
Las disciplinas de política social y pública forman parte integral de la educación en salud pública

Aumentan en prominencia, aunque Ciencias de la población y disciplinas todavía una parte relativamente menor de cuantitativas características esenciales del la formación 


\section{CONCLUSIÓN}

Cambios en los factores de morbilidad y mortalidad nos retan a reconsiderar los enfoques educativos de las escuelas de medicina y salud pública especialmente en el área de promoción de la salud y prevención de enfermedades. Los centros universitarios han avanzado en el trabajo en promoción de la salud y prevención de la enfermedad, dichos centros realizan esfuerzos acorde con los lineamientos de distintas cartas y conferencias de promoción de la salud.

El compromiso para repensar la promoción de la salud y prevención de manera renovada sigue vigente en los currículos de los programas de salud, y hay necesidad de replantear continuamente y de igual manera la educación recibida desde los currículos en promoción de la salud y prevención de enfermedades. La educación en salud se viene fortaleciendo como estrategia clave en la promoción de la salud y prevención de la enfermedad y el componente de comunicación en salud es clave para ésta y se ha venido fortaleciendo con los nuevos enfoques de la comunicación en salud.

Las especializaciones, maestrías y doctorados que se promueven y evolucionan en los distintas universidades crean un ambiente propicio para el espectro de intervenciones dirigidas al medio ambiente, el comportamiento humano, los estilos de vida y la atención médica, de hecho facilita el cambio de paradigma de atención en salud.

Queda aún brechas por acortar luego de pasar 3 décadas desde la denominada Carta de Otawa y la educación en salud puede contribuir a la disminución de oportunidades pérdidas medidas en enfermedades evitables, por lo tanto es un reto de las universidades e instituciones formadoras de recursos humanos que buscan cumplir propuestas comunes discutidas en las distintas conferencias de promoción de la salud y seguir apoyando las citadas recomendaciones de la Comisión de los determinantes sociales de la salud.

Financiamiento: Autofinanciado.

Conflicto de interés: Los autores declaran no tener conflictos de interés en la publicación de este artículo.

Recibido: 28 de noviembre

Aprobado: 30 de diciembre

\section{REFERENCIAS BIBLIOGRÁFICAS}

1.WHO. Promoción de la Salud: Glosario. 1998.

2. Pérez M, Pinzon-Perez H, Alonso Palacio L. Promoción de la salud: conceptos y aplicaciones. 1st ed. Barranquilla: Uninorte; 2007.

3. CDC. Achievements in Public Health, 1900-1999: Control of Infectious Diseases

[Internet]. Centers for Disease Control and Prevention. Centers for Disease Control and Prevention; [cited 12 Dec 2016]. Available from: https://www. cdc.gov/mmwR/preview/mmwrhtml/mm4829a1.htm

4. Department of Commerce and Labor, Bureau of the Census. Mortality Statistics, 1900 to 1904. Washington, DC: US Department of Commerce and Labor, 1906.

5. Hoyert DL, Kochanek KD, Murphy SL. Deaths: final data for 1997. Hyattsville, Maryland: US Department of Health and Human Services, Public Health Service, CDC, National Center for Health Statistics, 1999. (National vital statistics reports, vol 47, no. 19).

6. WHO. NCD mortality and morbidity [Internet]. World Health Organization. World Health Organization; [cited 8 Dec 2016]. Available from: http://www.who.int/gho/ncd/mortality_morbidity/en/

7. WHO. Noncommunicable Diseases [Internet]. World Health Organization. World Health Organization; [cited 8 Dec 2016]. Available from: http://www.who.int/mediacentre/factsheets/fs355/en/
8.WHO. The top 10 causes of death [Internet]. World Health Organization. World Health Organization; [cited 12 Dec 2016]. Available from: http:// www.who.int/mediacentre/factsheets/fs310/en/

9. NIH. How to Prevent and Control Coronary Heart Disease Risk Factors [Internet]. National Institutes of Health. U.S. Department of Health and Human Services; 2016 [cited 23 Jan 2017]. Available from: https://www. nhlbi.nih.gov/health/health-topics/topics/hd/prevent

10. Masuda J, Robinson K, Elliott S, Eyles J. Chronic Disease Prevention and the Politics of Scale: Lessons from Canadian Health Reform. Social Work In Public Health [serial on the Internet]. (2012, Nov), [cited February 3, 2017]; 27(7): 639-657.

11. WHO. Health promotion [Internet]. World Health Organization. World Health Organization; [cited 23 Jan 2017]. Available from: http://www. who.int/topics/health_promotion/en/

12. Organización Mundial de la Salud. Educación para la Salud: Manual sobre educación sanitaria en atención primaria de salud. Ginebra: OMS 1989.

13. Sanabria Ramos G. El debate en torno a la Promoción de Salud y la Educación para la Salud. Revista Cubana de Salud Pública. 2007; 33(2).

14. López S. Sistemas de salud comparados - Breve recorrido histórico y el impacto de las reformas de los años 90 [Internet]. [cited 23 Jan 2017]. Available from: http://www.monografias.com/trabajos34/sistemassalud/sistemas-salud.shtml 
15. Beldarrin E. La medicina social occidental. Revista Cubana Salud Pública. 2002; 28(1): 22-70.

16. WHO. Declaration of Alma-Ata. 1978. Available from: http://www. who.int/hpr/NPH/docs/declaration_almaata.pdf

17. Maceiras D. Carta de Ottawa para la promoción de la salud. Salud Pública y Educación en la Salud [Internet]. 2001; 1(1): 19-22.

18. Espinoza H. Participación social en salud: un reto para la promoción de la salud. Revista Facultad Nacional Salud Pública . 2003; 21(2): 119-26.

19. Forero Santos J. Participación social en salud: un reto para la promoción de la salud. Hacia Promoción de Salud. 2003; 8: 59-75.

20. Taipale V. Challenges in health promotion policies. IUHPE-Promotion and Education. 2002; 9(4): 143-6.

21. Report of the 2011 Joint Committee on Health Education and Promotion Terminology. American Journal of Health Education. 2012 43(2): 1-19

22. Caja Costarrisense de Servicio Social. Introducción a la Promoción de la Salud. San José, Costa Rica: Universidad de Costa Rica. 2004.

23. WHO. WHO | Health education [Internet]. WHO. World Health Organization; [cited 13 Dec 2016]. Available from: http://www.who.int/ topics/health_education/en/

24. GriffithsW. Health Education Definitions, Problems, and Philosophies. Health Education \& Behavior. 1972; 1(31): 7-11.

25. Yazachew, M., \& Alem, Y. Introduction to Health Education. Ethiopia: Jimma University. 2004.

26. Frank E, Hedgecock J, Elon L. Personal health promotion at US medical schools: a quantitative study and qualitative description of deans' and students' perceptions. BMC Medical Education. 2004; 4(1).
27. Gofin J., Gofin R. Atención primaria orientada a la comunidad: un modelo de salud pública en la atención primaria. Revista Panamericana de Salud Pública. 2007; 21(2-3): 177-185.

28. Franco Á. Atención Primaria en salud (APS)¿De regreso al pasado?. Revista Facultad Nacional de Salud Pública. 2015; 30(1): 83-94.

29. Ortiz P, Quintero E, Valdes C. ¿Por qé se debe renovar la atención primaria en salud?. Revista CES. 2013; 4: 77-81.

30. Hernández-Aguado I, Santaolaya Cesteros M, Campos Esteban P. Las desigualdades sociales en salud y la atención primaria. Informe SESPAS 2012. Gaceta Sanitaria. 2012; 26: 6-13.

31. Idler N, Huber J, von Mutius S, Welbergen L, Fischer M. Prevention and health promotion from theory to practice: The interprofessional MeMPE Summer University for students of Medicine, Master of Public Health and Epidemiology. GMS Journal For Medical Education [serial on the Internet]. (2016, Nov), [cited February 3, 2017]; 33(5): 1-8.

32. Macinko J, Montenegro $\mathrm{H}$, Nebot Adell C, Etiene C, Trabajo de Atención Primaria de Salud de la Organización Panamericana de la Salud. La renovación de la atención primaria de salud en las Américas. Rev Panam Salud Publica. 2007; 21(2-3): 73-84.

33. Giovanella L, Almeida P, Vega Romero R, Oliveira S, Tejerina Silva H. Panorama de la Atención Primaria de Salud en Suramérica: concepciones, componentes y desafíos. Saúde em Debate. 2015; 39(105): 300-322.

34. Harvard T.H. Chan. Public Health and Medicine: Distinctions Between Public Health and Medicine [Internet]. 2017 [cited 3 February 2017]. Available from: https://www.hsph.harvard.edu/about/publichealth-medicine/

Consulte la Versión Electrónica de la Revista:

Facultad de Medicina Humana Universidad Ricardo Palma

http://revistas.urp.edu.pe/ojs/index.php/RFMH<smiles>C1=[In]CC1</smiles>

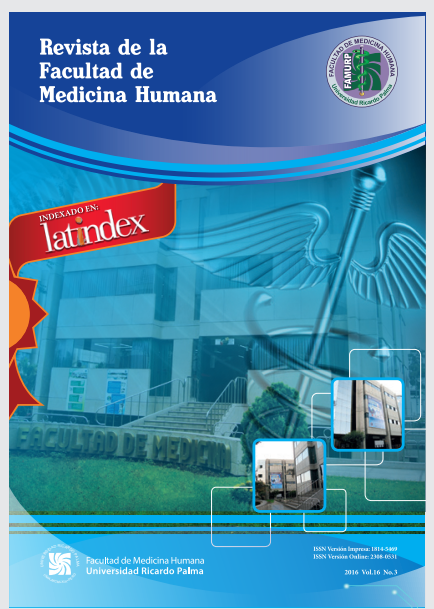

